EPJ Web of Conferences 70, 00069 (2014)

DOI: 10.1051/epjconf/ 20147000069

(C) Owned by the authors, published by EDP Sciences, 2014

\title{
International Particle Physics Masterclasses with LHC data
}

\author{
Panagiota Foka ${ }^{1}$ (on behalf of the International particle Physics Outreach Group - IPPOG) \\ ${ }^{1}$ GSI Helmholtzzentrum für Schwerionenforschung GmbH, Planck str. 1, 64291, Darmstadt, Germany
}

\begin{abstract}
The International Particle Physics Masterclasses is an educational activity developed by the International Particle Physics Outreach Group with the aim to bring the excitement of cutting-edge particle-physics research into the classroom. Since 2005, every year, thousands of pupils in many countries all over the world become "scientists for a day" in research centres or universities close to their schools as they are introduced to the mysteries of particle physics. In 2012, 10000 students from 148 institutions in 31 countries took part in this popular event over a month period. The program of a typical day includes lectures that give insight to topics and methods of fundamental research followed by a "hands-on" session where students perform measurements on real data from particle-physics experiments themselves. The last two years LHC data from the ALICE, ATLAS and CMS experiments were used. A overview of the performed measurements and the employed methodology is presented.
\end{abstract}

\section{Introduction}

The International Particle Physics Outreach Group (IPPOG) [1] is a network of scientists, researchers, science educators and communication specialists engaged in informal science education and outreach for particle physics. Within the framework of IPPOG an educational project, called International Masterclasses (IMC) [2], was developed with the aim of bringing experimental data and research methods to high-school students. The main idea was to give to students, aged 15-18, the possibility to experience how scientists investigate nature by doing it themselves. Universities and research centers invite students and their teachers from nearby schools and host them for a day. The students and their teachers get acquainted with topics and methods of basic research and on the fundamental laws of matter and forces. The main aim of the Masterclasses is to give them the possibility to perform measurements on real data from particle-physics experiments, as real scientists do, and the real highlight of the day is a "hands-on" session. The project started in 2005 with the analysis of real data from the DELPHI and OPAL experiments at the LEP collider at CERN, inspired by similar activities that were taking place in the UK.

As the experiments at the Large Hadron Collider (LHC) at CERN collect larger amounts of data, interesting new results are produced and are being published. Naturally, the wish is to share this excitement with the general public and in particular with school children, the potentially future generations of scientists. During the last two years, the International Masterclasses included measurements of a sample of LHC data from the ALICE, ATLAS and CMS experiments as detailed in [3] and [4]. Educational material, methods and analysis tools were developed for the analysis of the new data and the presentation of the analysis methods. Scientists, sharing the enthusiasm of our field,

This is an Open Access article distributed under the terms of the Creative Commons Attribution License 2.0, which permits unrestricted use, distribution, and reproduction in any medium, provided the original work is properly cited. 
explain to the teachers and young students how things really work through active participation and involvement in "do-it-yourself" measurements and searches.

\section{Typical Masterclass day}

Every year, a centrally organised event of the International Masterclasses runs during the month of March. Institutes and universities all over the world host for a day a class of high-school students. A maximum of five institutes participate during the same day doing the same measurement. At the end of the day all participating institutes join a video-conference to present and discuss their results. The video-conference is coordinated and moderated either by CERN or Fermilab, depending on the time zone.

The program of a typical day at each institute starts with introductory lectures on basic concepts of high-energy physics followed by a presentation of the relevant experiment. The aim is to give the students a flavour of research methods and not to rigorously teach them a physics course. First, the Standard Model is briefly explained summarizing our current understanding of the laws of nature while the open questions and remaining puzzles are outlined. The physics observables that scientists are looking for in order to complete the picture are discussed and the experiments designed to measure them are described. The students then visit a laboratory where some detector components for these experiments were constructed or when possible they visit the experiments themselves.

After a lunch break it is the time for the "hands-on" experience and the analysis of the LHC data. The students are brought into a computing room where computers have been setup for the specific measurement of the day. The tutor gives a brief explanation on how to use the software and tools to perform the specific measurement. The tutor also performs a real-life demonstration as an example. The students are then asked to proceed by themselves and analyse larger data samples, two of them working together in each computer, supervised and helped by tutors.

Students quickly master real event-display programs, software tools and analysis methods. They mostly come with the results within the allocated time. At the end, the tutor merges the results obtained by each group to increase statistics. Even with this simple procedure students realise that this type of research is not an one person's affair but requires collaborative effort. The tutor discusses the obtained results and their relevance to the physics questions exposed in the morning. The students prepare the final collective result of the classroom and a small presentation. They are then taken into a room with video-conferencing facilities and join a video-conference with CERN or Fermilab. All other participating institutes also join to compare and further discuss the obtained results. They use the same video-conferencing facilities and tools as scientists of our international research collaborations use to participate in common meetings with participants spread all over the world. They share their presentations, combine their results, discuss them and draw common conclusions. The resulting combinations are then compared to recent results published by the collaborations.

Several measurements are proposed to the participating institutes. The different measurements available for each one of the LHC experiments are detailed below. However, the basic ideas and aims behind them are similar. The students are first exposed to the methods of identifying particles that cross the different detectors by exploiting the characteristic signals left by particles in various subdetector elements; electrons, muons, photons and jets of particles can then be recognised. The students continue with selecting events based on specific signals. Using the different data samples, they measure different properties of some known particles, such as the $\mathrm{W}$ and $\mathrm{Z}$ weak gauge bosons and a number of hadrons $\left(\mathrm{J} / \psi, \mathrm{Y}, \Lambda, \mathrm{K}_{\mathrm{s}}\right)$.

Using the CMS and ATLAS data, they also study complex particle properties, such as the quarkgluon structure of the proton, through the fraction of $\mathrm{W}^{+}$and $\mathrm{W}^{-}$events, which they come to understand is not just the simple view of uud quarks. They also learn how the concept of invariant mass can be used to identify and measure masses and widths of short-lived particles. Furthermore, the invariant mass concept is applied to look for new particles. 
Finally, they are given the ingredients and methods to search for a new state of matter, a deconfined state of quarks and gluons, called quark-gluon plasma, which within the Standard Model Big-Bang theory is believed to have existed at the very early stages of the universe.

\section{Examples of measurements}

\subsection{ATLAS}

Two Masterclass measurements are proposed based on ATLAS data, the "Z-path" and "Wpath", developed by the Universities of Dresden and Oslo [5].

The "Z-path" [6] exploits the invariant mass concept as a tool used to identify known short-lived particles and to search for and discover new ones. An ATLAS data sample of 10000 events comprises 9000 real events (dileptons from Z, J/ $\psi, Y$, Drell-Yan, and other QCD and W data), and 1000 simulated dilepton $Z$ ' particles at a mass of $1 \mathrm{TeV}$. The event display software HYPATIA [7] is used for visual inspection of the events and particle identification. Each pair of students examines a subset of 50 mixed events. Based on this visual procedure, particles are identified, dilepton $\left(\mathrm{e}^{+} \mathrm{e}^{-}\right.$or $\left.\mu^{+} \mu^{-}\right)$ events selected and invariant masses calculated. A web-based application [8] provides a plotting tool as well as a facility to upload and combine the results. Figure 1 presents a typical invariant mass spectrum resulting from the dilepton data analysis. The students then discuss with the tutors details on the measured masses and widths of the known resonances, make a comparison between electrons and muons and finally compare with published results of ATLAS. Further investigation of the invariant mass spectrum leads to the "discovery" of a new particle with a mass around $1 \mathrm{TeV}$ - resulting in from the sample of the simulated Z' dileptons events combined with the real data. Such a "new" particle, a neutral gauge boson, is supposed to mediate a hypothetical "new" weak interaction supposedly predicted by certain theories beyond the Standard Model.

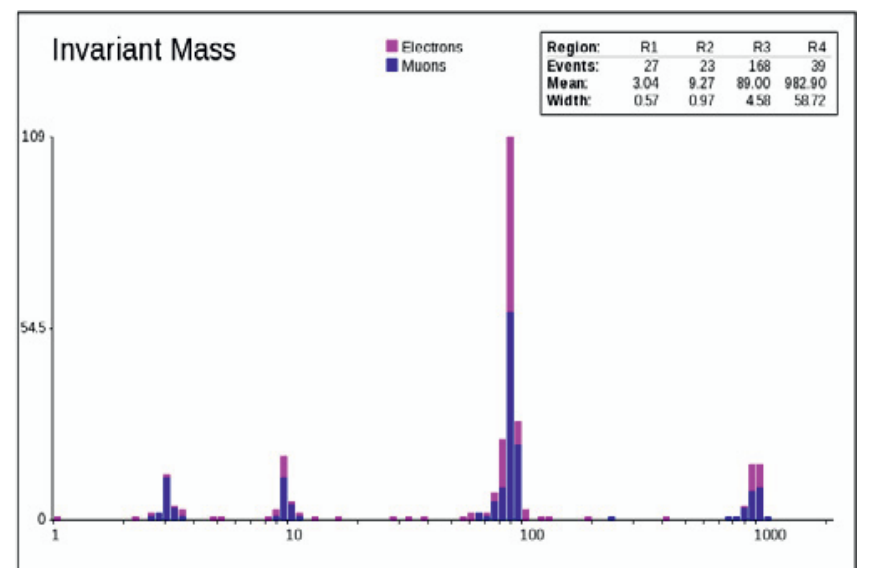

Fig. 1. ATLAS "Z-path" measurement: dilepton invariant mass spectrum as analysed by students, showing the $\mathrm{J} / \psi$, Y and $\mathrm{Z}$ resonances, from experimental data, as well as a "simulated" $\mathrm{Z}$ ' peak at $1 \mathrm{TeV}$ from simulated data including the "new" Z' particle.

The ATLAS "W-path" [9] makes use of a data sample of 6000 events, mainly consisting of $\mathrm{W} \rightarrow \mathrm{l} v$ events with a high transverse momentum electron or muon and some missing transverse energy/momentum. Some background events (QCD jets, Z, top) and 250 simulated $\mathrm{H} \rightarrow \mathrm{WW}$ are added. The event display program MINERVA [10] is used to identify $\mathrm{W}$ bosons. The students 
measure the fractions of $\mathrm{W}^{+}$and $\mathrm{W}^{-}$events and they plot the ratio $\mathrm{W}^{+} / \mathrm{W}^{-}$as shown in Figure 2 . This is then compared to the prediction they make using the simple uud model of the proton. They also measure the angle between leptons from two W-bosons in the transverse plane. The combined measurement of several groups is shown in Figure 3. To document their measurements students make use of online spread sheets [11] from which summary tables and histograms are then extracted. The data sample made available for student analyses has a $\mathrm{W}^{+} / \mathrm{W}^{-}$ratio of $1.56 \pm 0.17$, very close to the published ATLAS result of $1.52 \pm 0.07$.

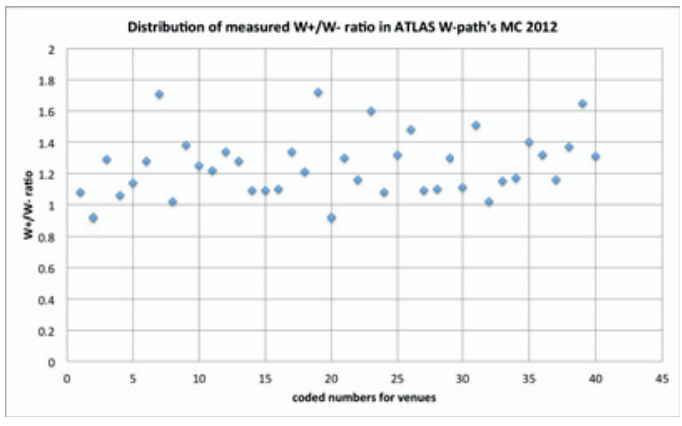

Fig. 2. ATLAS "W-path" measurement focusing on the study of the structure of the proton. $T$ The $\mathrm{W}^{+} / \mathrm{W}^{-}$ratio measured by the students is close to the one expected for this data sample, and far from the prediction they made using the simple $u u d$ model of the proton.

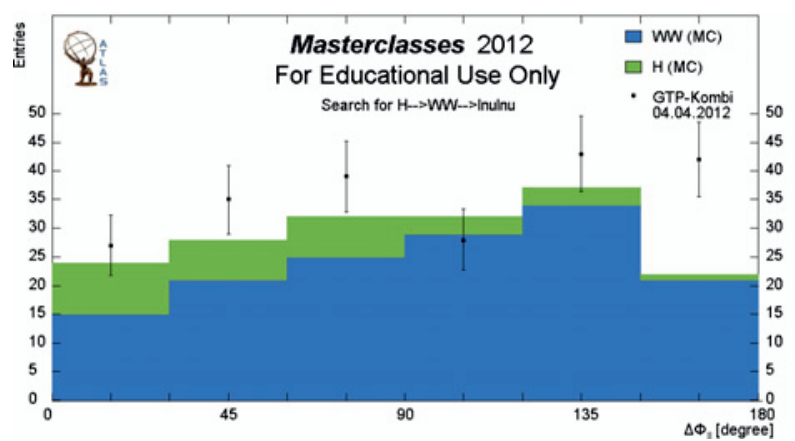

Fig. 3. ATLAS "W-path" measurement. The distribution of the angle between the two leptons, $\Delta \Phi_{11}$ in the transverse plane, as measured by the students, for WW (blue) and $\mathrm{H} \rightarrow \mathrm{WW}$ (green) events. The combined measurement of several groups of students is shown by "GTP-Kombi".

\subsection{CMS}

The measurements based on CMS data have mainly been developed by QuarkNet [12], I2U2 [13] and the CMS Education and Outreach team. In the 2012 version of the CMS Masterclass [14], students are asked to study $\mathrm{W}$ and $\mathrm{Z}$ boson candidates using the purely web-based event display software iSpy-online [15]. Students examine and manipulate events in three dimensions to determine lepton flavour (e or $\mu$ ), candidacy $\left(W, Z\right.$, or "zoo") and charge $\left(\mathrm{W}^{+}\right.$or $\left.\mathrm{W}^{-}\right)$. They then find $\mathrm{W}^{+} / \mathrm{W}^{-}$and $\mathrm{e} / \mu$ ratios, and reconstruct the $Z$-mass. They find that many of their $Z$ candidates were, in fact, other, lower mass particles such as $\mathrm{J} / \psi$ and $\mathrm{Y}$. The recording of events and calculations are done using an EditGrid web-based spreadsheet. An example of the measurement is shown in Figure 4. The charge determination using the event display is shown on the left and the resulting invariant mass distribution 
on the right. Students were able to determine that an event contains the decay of a W with $95 \%$ efficiency. From the correctly-identified events they were able to determine the charge (from electron or muon) for $92 \%$ of the events (if not, they marked them as "W candidate" with no charge specified). And for those events where they were able to determine the charge, they were able to determine it correctly with $96 \%$ efficiency. This high efficiency leads to the students finding a $\mathrm{W}^{+} / \mathrm{W}^{-}$ratio that is very close to the value in the data, which is in turn very close to the published value (1.43).

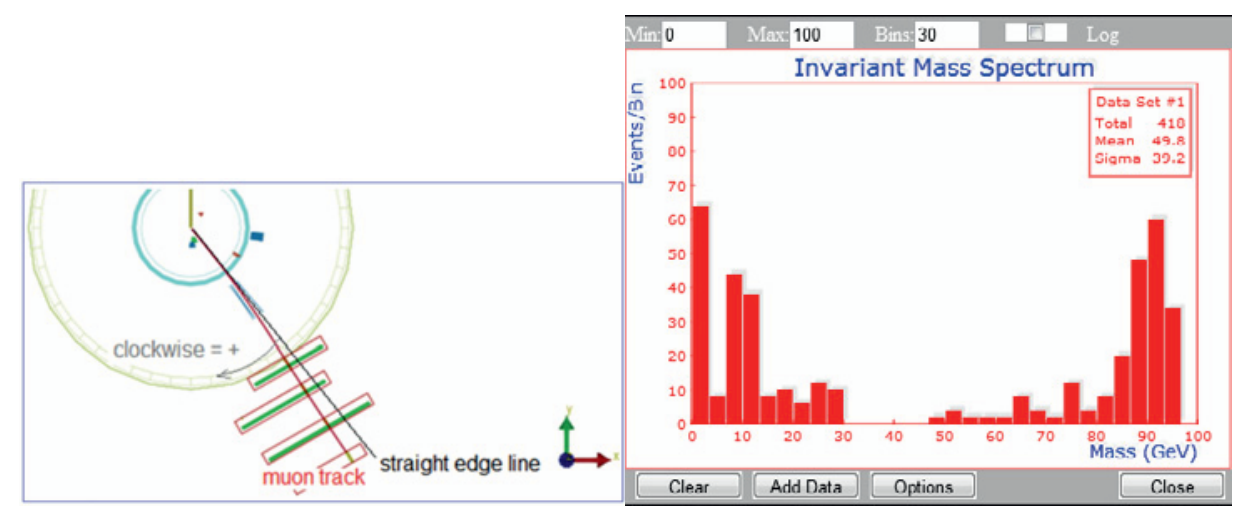

Fig. 4. Left: charge determination using the visual tools. Right: spectrum of resulting invariant mass.

\subsection{ALICE}

ALICE is studying the physics of heavy-ion collisions at the LHC with main emphasis on the search of a deconfined state of quarks and gluons, known as Quark-Gluon Plasma (QGP) and the characterisation of its properties. According to theoretical predictions normal hadronic matter undergoes a phase transition to the quark-gluon plasma above certain critical conditions of temperature and density. Experimentally it is verified that such conditions are reached and exceeded in head-on lead-lead collisions at the LHC.

In order to draw conclusions about the creation of the QGP and its properties, several observables have to be studied at the same time. Furthermore, such observables have to be compared to a baseline measurement and for that purpose experimental results from elementary proton-proton collisions are usually used. Alternatively one can compare to Monte-Carlo proton-proton results, which do not include modelling of quark-gluon plasma. One of the first predicted QGP signatures was enhanced production of "strange" particles relative to the one in pp collisions. On the other hand, at the high energies of the LHC high transverse momentum probes, like jets, become available. It is predicted that highly energetic jets of particles are attenuated when they cross the hot and dense medium produced in heavy-ion collisions, thereby providing information about its properties.

ALICE developed two Masterclass measurements, one based on the "strangeness enhancement" [16] and the other on the "jet quenching" [17] studies. The first one relies on the measurement of strange particles in $\mathrm{Pb}-\mathrm{Pb}$ collisions and their comparison to results from $\mathrm{pp}$ collisions. The later relies on the measurement of the transverse momentum spectra of unidentified particles in $\mathrm{Pb}-\mathrm{Pb}$ and $\mathrm{pp}$ collisions. Both observables are also studied as a function of centrality, which characterises the degree of the overlap of the two colliding nuclei. Head-on collisions provide most favourable conditions for the creation of QGP while the most peripheral collisions are similar to pp collisions.

During the 2012 ALICE Masterclass the students first visually inspected a small data sample of $\mathrm{pp}$ and $\mathrm{Pb}-\mathrm{Pb}$ data. They immediately witnessed the differences between $\mathrm{pp}$ and $\mathrm{Pb}-\mathrm{Pb}$ events. As the $\mathrm{Pb}-\mathrm{Pb}$ events are of much higher multiplicity, they are much more complex making the visual inspection difficult. The students also got acquainted with the concepts of tracking and particle 
identification, learning that particles bend in a magnetic field and what is the information one can extract from this. Strange particles decay after flying for some distance in the detectors leaving a characteristic decay pattern, known as $\mathrm{V}_{0}$ or cascade, which the students learn how to identify. By manipulating interactively the events in $3 \mathrm{D}$ they better appreciated the importance of precise pattern recognition and the concepts of background resulting from misidentified patterns. They also appreciated visual analysis as a means of better understanding the events and optimising selection criteria while realising its limitations for the analysis of large statistics.

For the Masterclass a simplified version of the standard ALICE event display was developed which is based only on ROOT [18] and is independent on any other software of ALICE. Thanks to its functionality it allows filling of histograms and tables within the same environment [19] in addition to the visual inspection of the events. A snapshot of the "strangeness enhancement" analysis tool is shown in Figure 5. The tool includes $\mathrm{V}_{0}$ and cascade decay finders and allows the calculation of invariant masses of different species of strange particles as well as filling of the corresponding histograms. It also implements methods to analyse large statistics samples of pp and $\mathrm{Pb}-\mathrm{Pb}$ events in the same environment used for the visual inspection of individual events. A fitting procedure was implemented allowing the students to choose the limits for the fit, fit separately the background and the peak of the signal and then subtract them to obtain the number of strange particles. Finally, the results obtained from the analysis of the pp data samples are compared to Monte-Carlo calculations and to the published results of the experiment. For the next edition of the measurement, $\mathrm{Pb}-\mathrm{Pb}$ data of different centralities will be analysed. The goal is, by comparing results from $\mathrm{pp}$ and $\mathrm{Pb}-\mathrm{Pb}$ collisions, to observe strangeness enhancement as a tell-tale signal of the production of the quark-gluon plasma.

The "jet quenching" measurement implements the analysis of large statistics $\mathrm{Pb}-\mathrm{Pb}$ samples of different centralities via a macro and produces the transverse momentum spectra of unidentified particles for different centralities. Those, properly normalised, are divided by the pp spectrum. Figure 6 shows the resulting spectrum, which quantifies the suppression and is known as nuclear modification factor, $\mathrm{R}_{\mathrm{AA}}$. Finally, the obtained results are compared to the published data, which were among the ALICE "first day measurements". A huge suppression, factor five, is observed for the most central events, manifesting the "jet quenching", which suggests that particles lose energy traversing a very dense medium as expected for the quark-gluon plasma.

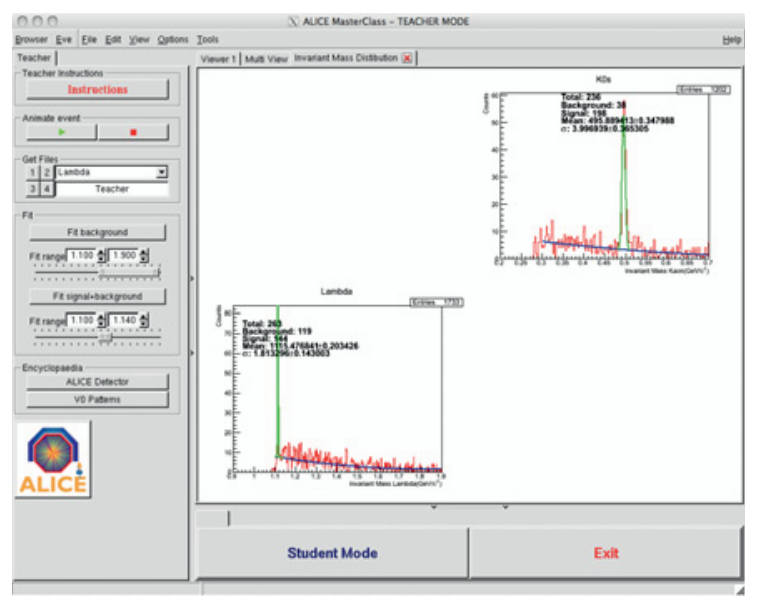

Fig. 5. Snapsot of the analysis tool used for the ALICE "strangeness enhancement" measurement. The same tool produces the invariant mass plot for $\mathrm{K}_{\mathrm{s}}$ and $\Lambda$, shown fitted using different options provided by sliders. 


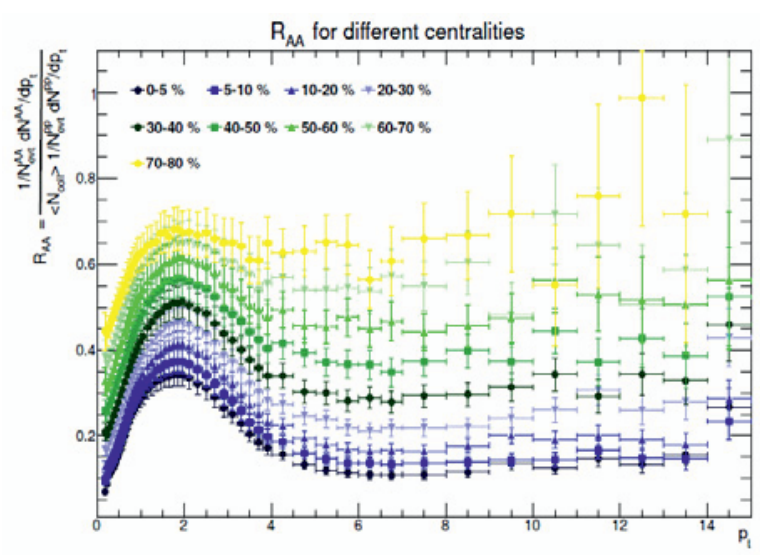

Fig. 6. ALICE "jet quenching” measurement: $\mathrm{R}_{\mathrm{AA}}$ of charged particles produced in $\mathrm{Pb}-\mathrm{Pb}$ collisions for different centralities. A large suppression is observed for the most central, "head-on" collisions at the high transverse momentum regime, reaching up to $15 \mathrm{GeV} / \mathrm{c}$ with the sample used for the Masterclass. The shape of the spectrum and suppression factor are very close to the ALICE published results.

\section{Statistics}

The Masterclasses based on measurements of the LHC data were first introduced in 2011. Compared to 2011 statistics, an overall increase in participation to the 2012 IPPOG Masterclasses was seen, resulting in an increase in the number of days that they were performed. Table 1 presents details on the number of countries, institutes and students that participated as well as the number of classes, video-conferences and moderators that assisted the video-conferences. Table 2 presents the breakdown of the specific performed measurements.

\begin{tabular}{|l|c|c|c|c|c|c|c|}
\hline & Days & Countries & Institutes & Students & Moderators & VC & Classes \\
\hline 2011 & 19 & 26 & 99 & 9000 & 15 & 23 & 116 \\
\hline 2012 & 21 & 31 & 118 & 10000 & 21 & 36 & 143 \\
\hline
\end{tabular}

Tab. 1. IMC statistics in 2011 and 2012. VC stands for video-conferences at CERN or Fermilab led by moderators.

\begin{tabular}{|l|c|c|c|c|}
\hline & ALICE & ATLAS W & ATLAS Z & CMS \\
\hline 2011 & 10 & 49 & 31 & 26 \\
\hline 2012 & 13 & 42 & 53 & 35 \\
\hline
\end{tabular}

Tab. 2. Break-down of IMC statistics on LHC measurements. In addition, 31 institutes followed the 2012 US program; 13 did the ATLAS Z-measurement and 18 the CMS W\&Z measurement.

Immediately after the Masterclasses surveys are conducted to assess the whole event allowing the evaluation of different aspects. They provide information on needed improvements and details of topics that have to be introduced or simplified. The results of the 2012 surveys have shown that the IPPOG Masterclasses were a great success. They also show that students, teachers and moderators enjoy the event and that the interest in participation is growing. After the Masterclasses students have an increased interest in basic research and their participation results in expanding their views and learning many things. However, the surveys also show that there is still room for 
improvements. In particular students find that the theory is sometimes rather difficult to grasp. On the other hand some hands-on measurements have the tendency to be too easy and repetitive. Some students ask for more challenges and to be updated on the research front. They wish to "take part in discoveries".

\section{Prospects}

The map shown in Figure 7 gives a visual impression of the countries that participated in the 2012 International Masterclasses and their spread all over the world. For the 2013 Masterclasses, at least eight more countries have expressed interest to join and will be added to the 2012 map below: Australia, Cyprus, Georgia, India, Turkey, Romania, Egypt, Palestine.

In addition to the International Masterclasses event, organised centrally in March, further Masterclasses are also organised "on-demand" when and where it suits, for events such as the "teacher's day", "women's day", to mention few examples. Furthermore, tutors visit schools and perform the measurements in the student's classroom usually using the Masterclass material installed in laptops.

Every year, all measurements are being updated and improved incorporating new material and expanding the scope. Continuous effort is invested in improving the existing tools with the aim to facilitate the work of tutors, and make the measurements easier for the students. The aim is to present uniform packages for all measurements, to the level that it is possible, in order to lessen the overhead of the practical issues related to the tools and documentation. Therefore, work is ongoing with the aim to converge towards common tools for plotting, uploading, combining the results and looping over large samples of data. Moreover, effort is invested on the documentation, which is also becoming more detailed and systematized in a uniform way for all measurements.

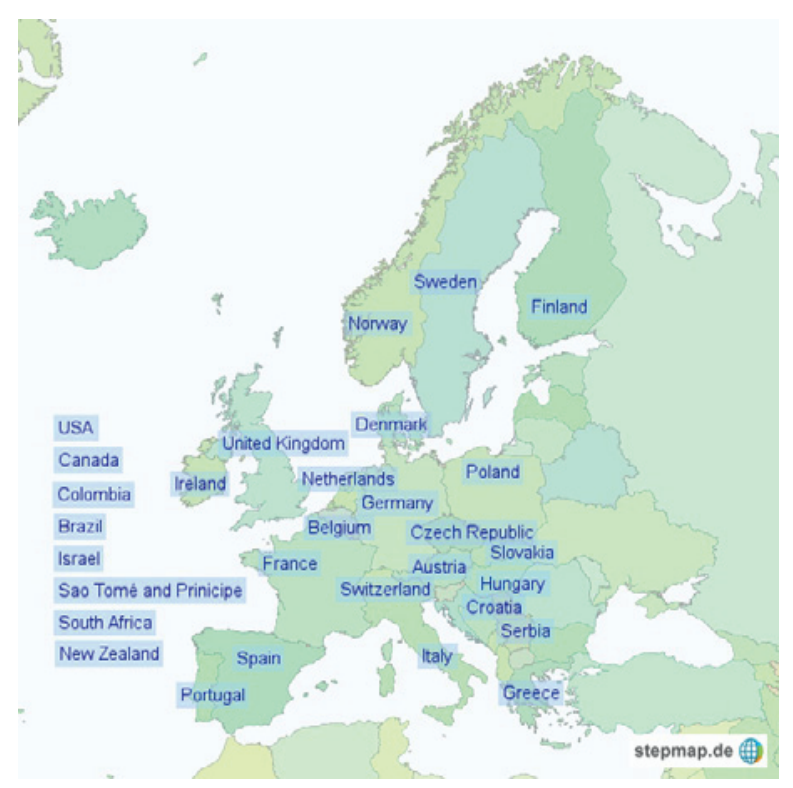

Fig. 7. Countries that participate in the International Masterclasses 2012.

Also, every year, the data samples are updated and enriched. The LHC experiments, recognizing the success and potential of the International Masterclasses, have recently approved the release of larger and more exotic data samples. The ATLAS collaboration makes available $1 \mathrm{fb}^{-1}$ of data for the use of the Masterclasses to cover "Higgs searches". The Z-path is extended to apply the invariant 
mass technique with the aim to cover current research highlights such as $\mathrm{H} \rightarrow \mathrm{ZZ} \rightarrow 1111$ and $\mathrm{H} \rightarrow \gamma \gamma$. The W-path will make use of real WW events. The CMS collaboration makes available several Higgs candidates in the mass region of interest in various decay channels for "treasure hunt" activities. In the next version of the ALICE measurements, large scale analysis of lead-lead collision data will be used more extensively.

An extensive collection of resources [20] can be found in the IPPOG web site. They are intended to provide guidelines and material for particle physicists that wish either to get involved in this activity or to develop their own initiatives. Further Masterclass activities can be developed based on the samples of the LHC data using the material provided in the web pages and the associated tools. To keep the interest of the students vivid new ideas and concepts are highly welcome.

\section{References}

1. International Particle Physics Outreach Group - IPPOG, http://ippog.web.cern.ch, http://facebook.com/IPPOG

2. International Masterclasses, www.physicsmasterclasses.org

3. F. Ould-Saada, International Particle Physics Masterclasses - Bringing LHC data in the Classroom (to be published in ICHEP 2012 Proceedings)

4. P. Foka, International Particle Physics Masterclasses (to be published in CONFINEMENT X Proceedings)

5. LHC@InternationalMasterclasses, http://atlas.physicsmasterclasses.org/en/index.htm

6. M. Pedersen, F. Ould-Saada, E. Gramstad, M. Bugge, University of Oslo, The ATLAS Zpath, http://atlas.physicsmasterclasses.org/en/zpath.htm

7. HYPATIA, Ch. Kourkoumelis, D. Fassouliotis, S. Vourakis (University of Athens), D. Vudragovic (Institute of Physics, Belgrade), http://hypatia.phys.uoa.gr

8. V. Morisbak, M. Pedersen, F. Ould-Saada, University of Oslo, OPloT, http://cernmasterclass.uio.no/OPloT/index.php

9. K. Jende, M. Kobel, G. Pospiech, U. Bilow, C. Rudolph, University of Dresden, The ATLAS W-path, http://atlas.physicsmasterclasses.org/en/wpath.htm

10. MINERVA, T. McLaughlan, M. Stockton, P. Watkins (University of Birmingham), M. Wielers (Rutherford Appleton Laboratory), http://atlas-minerva.web.cern.ch/atlas-minerva

11. EditGrid, http://www.editgrid.com/user/masterclass/Analysis_2012

12. QuarkNet, http://quarknet.fnal.gov

13. I2U2, http://www.i2u2.org

14. K. Cecire et al, CMS Masterclass W/Z Measurement

15. iSpy-online, M. Hategan, P. Nguyen, T. McCauley, i2 $\mathrm{u} 2$ and QuarkNet collaborations, http://www.i2u2.org/elab/cms/event-display

16. P.R. Debski, P.Foka, D.Hatzifotiadou, B.Hippolyte, A. Maire, M. Tadel. Proceedings of the 7 th International Conference Hands-on Science Bridging the Science and Society gap, July 25 - 31, 2010, Rethymno - Greece.

http://aliceinfo.cern.ch/public/MasterCL/MasterClassWebpage.html

17. $\mathrm{R}_{\mathrm{AA}}$ Masterclass, http://www-alice.gsi.de/masterclass/

18. ROOT, http://root.cern.ch/

19. AliEve http://aliweb.cern.ch/Offline/Activities/Visualisation/index.html

20. IPPOG database, http://ippog.web.cern.ch/resources 